than that of the surrounding crust; and even a small deviation from the axis can produce a significant effect here because the depth age curve close to a spreading centre is steep. Then for a ridge formed subaerially, the amount of subsidence occurring before the ridge top sank below sea level will not be recorded in the measured subsidence. The net result of all such effects will be to produce a scatter of points along the depth-age curve and also to bias the points towards too small a measured subsidence.

In the circumstances, then, it is perhaps surprising that the difference between the measured subsidence and the subsidence predicted from the behaviour of normal crust is generally no more than $300-400 \mathrm{~m}$. However, such relatively small deviations apply only to the five ridges under consideration; other aseismic ridges conform much less well. For example, the IcelandFaroe Ridge evidently formed above sea level and remained that way for at least $15 \mathrm{Myr}$ before beginning its subsidence. On the other hand, Coiba Ridge, Carnegie Ridge and Cocos Ridge (Pacific) seem to have formed well below sea level and are thus now unusually deep. Then again, Madagascar Ridge, Broken Ridge and the Naturaliste Plateau (Indian Ocean) seem to have complicated histories of subsidence and uplift.

In view of these exceptions, it seems that the Detrick model can make no claim to widespread application. Yet there is more to it than that. It could be said, at least as an hypothesis, that the model applies generally to all aseismic ridges that form at spreading centres and at sea level. It does not necessarily follow from this, however, that all aseismic ridges are formed at spreading centres and at sea level. What may be true nevertheless is that, whereever a ridge forms, when it subsides it does so at a rate comparable to that of normal oceanic crust and as a direct result of the cooling and contraction of the lithosphere.

\section{New twists in bacterial flagella}

from Michael Spencer

Since the last comment in these columns (Nature, 263, 370; 1976) more light has been shed on a number of problems relating to the structure of bacterial flagella. The first is the geometrical puzzle set by bacteria having more than one flagellum: if all are helices undergoing real or apparent rotation, can they avoid tangling together? It is a simple matter to test this empirically by twiddling two pieces of wire between the finger and thumb, but a rigorous analysis is more daunting. Macnab (Proc. natn. Acad. Sci. U.S.A. $74,221 ; 1977)$ has now tackled the problem using geometrical and hydrodynamic theory, assisted by working models.

His conclusions confirm the fingerand-thumb analysis, showing that if a group of left-handed helices starts to rotate in a counterclockwise sense when viewed looking towards the basal points of attachment, the flagella are constrained to rotate in phase with each other and will continue to do so without hindrance; they do, however, undergo some distortion so as to form a compact bundle. This is what is observed in the normal swimming of bacteria. When the direction of rotation reverses, however, the helices should rapidly interlock so as to prevent further rotation (unless the flagella are very short, as occurs in some species). If both the handedness of the helix and the sense of rotation are reversed, jamming is not expected and normal forward motion can be resumed, with the flagella pointing to the rear.

Now comes the surprising part. Macnab and Ornston (J. molec. Biol. 112,1 ; 1977) report that a bacterium does not simply stop when the flagella reverse their rotation: the bundle flies apart, and the clockwise-rotating flagella are observed to have the smaller pitch characteristic of the 'curly' mutant. As first shown by Shimada et al. (Nature 254, 332; 1975), this has a right-handed helix. If the process is slowed down by irradiating with blue light in a high-viscosity medium these flagella sometimes form a curly bundle, giving forward motion again. Normally, however, there is a brief period of 'tumbling' followed by reversion to the previous state: a bundle of left-handed helices is reformed and the organism swims off in a new direction.

This periodic change of direction is an essential part of the chemotactic response, for external stimuli seem to influence the probability of tumbling: movement in a favourable direction suppresses the urge to tumble. This applies whether the bacteria are moving towards an attractant or away from a repellant, and must involve some kind of memory mechanism linked to the receptors (Macnab \& Koshland J. molec. Biol. 84, 399; 1974). The observed change of helix parameters seems to be essential, for without it the bacteria might simply stop and start again without changing direction, and there would be no point in reversing the sense of rotation. Only in certain special cases can backward motion be sustained.

The obvious question is "What makes the helix change when rotation is reversed?" After studying a number of mutant forms and the effects of abnormal growth media, Macnab and Ornston conclude that the torsional stress arising from a reversal of rotation actually induces a change in the packing of the flagellar subunits, so that the structure flips from a lefthanded to a right-handed helix. On removal of the stress, the structure clicks back into its normal shape. Their paper includes a number of photographs in which the transition is caught propagating out from the base of the flagellum, where torsion is expected to be greatest. As confirmation of this idea, abnormal curly flagella are observed to flip into the normal form under stress - the reverse of the normal process.

An outstanding problem-apart from the key question of how rotation is generated-is the nature of the forces that hold the subunits of a flagellum together. Kamiya and Asakura (J. molec. Biol. 108, 513; 1977) have some new evidence which supports the idea that electrostatic charge interactions are important. It has been known for some time that reconstituted flagella from Salmonella can change from the left-handed normal helix to the right-handed curly form on reduction of the $p \mathrm{H}$. Kamiya and Asakura now report that the same transition can be induced by an increase of $p \mathrm{H}$ above neutrality. In this case, as for the acidic transition, the curly form is attained by passing through an intermediate ('coiled') form. It can hardly be a coincidence that this sequence of transitions is just what would be expected from a geometrical packing model of the kind suggested by Calladine ( $J$. theor. Biol. 57, 469; 1976). It looks as if the ionisation induced by extremes of $p \mathrm{H}$ causes longitudinal rows of subunits to rearrange themselves, causing a discrete change in the helix parameters for each row thus transformed. Calladine's working hypothesis of a 'hinge' between subunits, giving two alternative arrangements, is supported by several biological examples; the latest to be described is tomato bushy stunt virus (Winkler et al. Nature, 265, 509; 1977).

This latest work on flagella illustrates how electrostatic and mechanical forces can separately induce a similar structural change. Macnab and Ornston make the point that a stress-induced transition may be relevant in other systems having regularly-arranged subunits. Who knows, at present, whether it might not be at the heart of muscular contraction? But that is another story. 